\title{
X-RAY NANO-DIFFRACTION ON EPITAXIAL CRYSTALS
}

Mojmír Meduňa ${ }^{1,5}$, Claudiu V. Falub ${ }^{2, \#}$, Fabio Isa ${ }^{3}$, Daniel Chrastina ${ }^{3}$, Thomas

Kreiliger $^{2}$, Giovanni Isella ${ }^{3}$, Alfonso G. Taboada ${ }^{2}$, Philippe Niedermann ${ }^{4}$, and Hans von Känel ${ }^{2, *}$

${ }^{1}$ Department of Condensed Matter Physics, Masaryk University, Kotlářská2,CZ-1137 Brno, Czech Republic

${ }^{2}$ Laboratory for Solid State Physics, ETH Zurich, CH-8093 Zurich, Switzerland

${ }^{3}$ L-NESS, Dip. di Fisica del Politecnico di Milano, Via Anzani 42, I-22100 Como, Italy

${ }^{4}$ CSEM, Jaquet-Droz 1, CH-2002 Neuchâtel, Switzerland

${ }^{5}$ CEITEC, Masaryk University, Kamenice 5, CZ-60177 Brno, Czech Republic

${ }^{\#}$ Present address: OC Oerlikon Advanced Technologies, Iramali 18, 9496 Balzers, Liechtenstein

* Corresponding author

Mailing address:

Laboratorium für Festkörperphysik, ETH Zürich

Schafmattstrasse 16

CH-8093 Zürich, Switzerland

Phone: +41 446332261

FAX: +41 446331072

e-mail: vkaenel@solid.phys.ethz.ch 


\begin{abstract}
The concept of growing epitaxial Ge and SiGe crystals onto tall Si pillars may provide a means for solving the problems associated with lattice parameter and thermal expansion coefficient mismatch, i.e., dislocations, wafer bowing and cracks. For carefully tuned epitaxial growth conditions the lateral expansion of crystals stops once nearest neighbors get sufficiently close. We have carried out scanning nano-diffraction experiments at the ID01 beam-line of the European Synchrotron Radiation Facility (ESRF) in Grenoble on the resulting space-filling arrays of micron-sized crystals to assess their structural properties and crystal quality. Elastic relaxation of the thermal strain causes lattice bending close to the Si interface, while the dislocation network is responsible for minute tilts of the crystals as a whole. To exclude any interference from nearest neighbors, individual Ge crystals were isolated first by chemical etching followed by micro-manipulation inside a scanning electron microscope. This permitted us to scan an X-ray beam, focused to a spot a few hundreds of nm in size, along the height of a single crystal and to record three-dimensional reciprocal space maps at chosen heights. The resolution limited width of the scattered X-ray beams reveals that the epitaxial structures evolve into perfect single crystals sufficiently far away from the heavily dislocated interface.
\end{abstract}




\section{Introduction}

The monolithic integration of photonics with Si electronics is one of the most intriguing examples in which silicon has to be combined with group III- $V^{1,2}$ or other group IV ${ }^{3,4}$ materials. Most of these are characterized by significantly different lattice parameters and thermal properties with respect to the Si substrate. These differences, and the way in which they induce defect formation when two mismatched semiconductor crystal lattices are epitaxially joined, have continued to form a topic of scientific interest and practical relevance ever since the pioneering work of Matthews and co-workers more than four decades ago ${ }^{5}$. Misfit dislocations (MDs) inevitably form at a film/substrate interface when the thickness of a mismatched layer exceeds a certain critical value, at which it becomes energetically favorable to release the misfit strain. However, they hardly affect an active region of a film, which is far above the interface region. Since dislocations have to end on defects, MD segments are, however, always connected to threading arms which necessarily extend to the surface unless they form closed loops. The more of these threading dislocations (TDs) pierce through the active region of a device, the more its properties deteriorate. It is therefore of utmost importance to keep the densities of TDs as low as possible.

Many methods devised over the years have been more or less successful in reducing the density of TDs, such as strained-layer superlattice formation ${ }^{6}$, compositional grading ${ }^{7,8}$, intentional introduction of point defects followed by thermal annealing ${ }^{9-11}$, and limited-area

growth. The latter has been accomplished either by substrate patterning ${ }^{12-14}$ or by selective area deposition (SAD) into dielectric windows present on a flat substrate ${ }^{15-20}$. In crystals with the zinc-blende and diamond structures the misfit strain is released mainly by $60^{\circ}$ 
dislocations for misfits below $2 \%$,2,6,21 Their threading arms lie in $\{111\}$ glide planes and can reach the sidewalls of structures on (001)-oriented substrates with high enough aspect ratios (height to width). Using SAD in this way has been called "epitaxial necking"18 or “aspect ratio trapping" (ART) ${ }^{19}$. While ART is very efficient in removing $60^{\circ}$-dislocations, it does not eliminate so-called "growth dislocations" which are oriented along the growth direction $^{22,23}$, unless it is combined with surface faceting. The expulsion of TDs by surface faceting is a result of their tendency to bend into directions perpendicular to a facet ${ }^{23,24,25}$. While most scientific attention has been paid in the past to TDs caused by the lattice parameter mismatch, unequal thermal expansion coefficients of epitaxial layers and substrates may have equally dire consequences for certain device applications, since it may result in layer cracking ${ }^{26}$ and wafer bowing ${ }^{27}$. Device processing may thus be impossible, or device yield may be very low.

Recently, we have developed a method of growing almost arbitrarily thick Ge structures on $\mathrm{Si}(001)$ substrates without any TD reaching the top of the crystal, cracks or significant wafer bowing, despite a lattice parameter mismatch of $4.2 \%$ and a mismatch of thermal expansion coefficients of $130 \%^{288}$. The method is based on deep substrate patterning at a micron scale, combined with growth conditions carefully tuned in order to limit lateral growth. This results in a space-filling array of three-dimensional Ge crystals up to $50 \mu \mathrm{m}$ in height. In this paper we shall focus on the detailed structural investigation of these microcrystals by X-ray nano-diffraction using synchrotron radiation and show how it compares with standard high-resolution measurements carried out with a laboratory source. We analyze how strain relaxation, crystal tilts and crystal perfection vary as a function of the distance from the heavily defective $\mathrm{Ge} / \mathrm{Si}(001)$ interface. 


\section{Experimental details}

Four inch, (001)-oriented (within $\pm 0.5^{\circ}$ ) $\mathrm{Si}$ substrates were patterned into Si pillars by conventional photolithography and deep reactive ion etching (DRIE). The pillars were 8$16 \mu \mathrm{m}$ high, had a base width of $2 \mu \mathrm{m}$, and were spaced either uniformly at separations between 1 and $4 \mu \mathrm{m}$ or in blocks of $10 \times 10$, the blocks being separated by $2.5 \mu \mathrm{m}$ wide trenches.

Space-filling arrays of closely spaced, tall Ge crystals were grown by low-energy plasmaenhanced chemical vapor deposition (LEPECVD) ) $^{299}$ at a rate of $\sim 4 \mathrm{~nm} / \mathrm{s}$ and at substrate temperatures between $400^{\circ} \mathrm{C}$ and $600^{\circ} \mathrm{C}$, using pure germane $\left(\mathrm{GeH}_{4}\right)$ as a precursor gas. In order to isolate some Ge crystals for the nano-diffraction experiments, the samples were first etched in a $30 \mathrm{wt} \% \mathrm{H}_{2} \mathrm{O}_{2}$ solution $\left(90 \mathrm{sec}\right.$ at $70^{\circ} \mathrm{C}$ ) to increase the gap between nearest neighbors. Subsequently, the crystals surrounding the one to be isolated were removed by micromanipulators inside a scanning electron microscope (SEM). Reference marks were written with a focused ion beam (FIB) in the vicinity of the isolated Ge crystals in order to enable their identification on the synchrotron diffractometer stage.

Prior to the synchrotron nano-diffraction experiments, the crystalline quality and strain of the Ge crystals were investigated by conventional high-resolution X-ray diffraction (XRD), with $\mathrm{Cu} \mathrm{K} \alpha_{1}$ radiation using a PANalytical X'Pert Pro-MRD diffractometer equipped with a 4-bounce $\mathrm{Ge}(220)$ crystal monochromator on the incident beam and an analyzer crystal and a Xe point detector on the diffracted beam. The X-ray beam diameter on the sample was $\sim 1 \mathrm{~mm}$. Therefore, depending on the lateral dimensions of the pattern, several thousand Ge crystals were simultaneously probed. 
In order to map the strain status locally, we performed X-ray experiments at the ID01 beamline of the European Synchrotron Radiation Facility (ESRF), using an $11.07 \mathrm{keV}$ beam focused down to $\sim 300 \times 500 \mathrm{~nm}$ by means of Fresnel zone plates (FZP). The structures were mounted within a Huber diffractometer equipped with a high-precision $(x, y, z)$ piezostage. This piezo-stage was used to scan the X-ray beam across the sample for a range of incidence angles $\omega$ on either side of the symmetric (004) and asymmetric (115) Bragg peaks. ${ }^{30}$ The use of a two-dimensional (2D) pixel detector permitted three-dimensional (3D) reciprocal space maps (RSMs) to be constructed for each $(x, y)$ position of the X-ray beam. These 3D RSMs were built from rocking scans, i.e., by varying the incidence angle $\omega$ of the primary focused beam.

\section{Results}

\section{(1) Laboratory diffractometer}

An example of RSMs recorded with the conventional laboratory diffractometer around the $\operatorname{Si}(004)$ and $\operatorname{Si}(224)$ reflections is shown in Fig. 1(a). The measurements were taken on arrays of $16 \mu \mathrm{m}$ tall, closely spaced Ge crystals grown onto $2 \mu \mathrm{m}$ wide Si pillars spaced either $2 \mu \mathrm{m}$ apart and arranged in blocks of $10 \times 10$ separated by $2.5 \mu \mathrm{m}$ wide trenches, or uniformly spaced $4 \mu \mathrm{m}$ apart. An SEM image of the region between two blocks can be seen in Fig. 1(b). The Si pillars can better be seen in the SEM image of Fig. 1(c), showing a uniformly spaced array after etching in $\mathrm{H}_{2} \mathrm{O}_{2}$, as a result of which the spacing between the Ge crystals is even more enlarged. The $Q_{z}$ coordinate in Fig. 1(a) is taken along the [001] surface normal and the $Q_{x}$ coordinate along [110]. The (004) and (224) diffraction spots 
expected for a Ge crystal strained to the Si lattice parameter are indicated by filled circles in the lower part of Fig. 1(c). In the absence of crystal tilt they appear at exactly the same $Q_{x}$ values as the corresponding $\mathrm{Si}$ reflections. The inclined dashed line through the $\mathrm{Si}(224)$ diffraction spot passes through the origin of reciprocal space. The (224) reflection of any cubic crystal with the same orientation as the Si substrate must necessarily appear on this line. As can be seen, this is clearly the case for the $\mathrm{Ge}(224)$ reflection. This, together with the fact that the maximum of the $\mathrm{Ge}(004)$ reflection appears at the same $Q_{z}$ value, proves that the $16 \mu \mathrm{m}$ tall Ge crystals studied in the measurement of Fig. 1(a) are completely strain-free. Now it can be safely assumed that at the growth temperature the misfit strain of a $16 \mu \mathrm{m}$ thick Ge-layer is fully relaxed. If a strain were present at room temperature at which the measurement was taken, it would necessarily have its origin in the different thermal expansion coefficients of $\mathrm{Ge}$ and $\mathrm{Si}$. Since no such strain has been measured, it must have been released elastically during cooling the sample to room temperature.

According to Fig. 1(a) the $\mathrm{Ge}(004)$ and $\mathrm{Ge}(224)$ diffraction spots are both elongated in the direction perpendicular to the scattering vector. In thin films such a broadening can be traced to the presence of dislocations, leading to mosaic spread ${ }^{31}$. Interestingly, in our case the diffraction spots are very narrow in the direction of the scattering vector, in fact as narrow as those of a defect-free Ge wafer ${ }^{288}$. For better visibility, we display an enlarged part of the RSM around the $\mathrm{Ge}(004)$ reflection in Fig. 2, together with that obtained from a planar region on the same wafer. As noted before, the main scattering intensity appears at the $Q_{z}$ value of fully relaxed and hence cubic Ge. The spot is, however, elongated along $Q_{x}$, with a maximum $I$ at $Q_{x}=0$ and two side maxima $I I$ farther out on the $Q_{x}$ axis. These side maxima can tentatively been attributed to the outermost row of Ge crystals in a $10 \times 10$ 
block which appear to be slightly asymmetric as seen in Fig 1(b) ${ }^{28}$. As will become clear from the synchrotron nano-diffraction experiments discussed below, the broadening of diffraction spots in the direction perpendicular to the scattering vector in Fig. 1 and Fig. 2 is indeed due to a random individual tilt of the Ge crystals rather than to mosaic spread caused by dislocations.

Finally, there are intensity maxima $I I I$ and $I V$ from patterned and planar regions, respectively, which appear exactly at the same $Q_{z}$ for both. These must be attributed to the tensile strain of $0.14 \%$ which develops during cooling from the growth temperature of $440^{\circ} \mathrm{C}$ to room temperature at which the $\mathrm{XRD}$ measurement is performed. In planar material the intensity of diffraction spot $I V$ is evidently strong since it is the only component present. On the other hand, for the patterned part of the sample the corresponding peak III is weak, since it can only stem from strained material at the bottom of the Si trenches, and therefore is greatly attenuated. For the $\mathrm{Ge}(224)$ reflection this peak is not observed at all, because it is measured at a grazing incidence angle of $\omega \sim 6.6^{\circ}$ for which the attenuation is complete.

\section{(2) Synchrotron nano-diffraction}

According to the laboratory diffraction experiments, the space filling Ge-crystals are relaxed but exhibit a mosaicity, the origin of which can only be unambiguously identified by synchrotron nano-diffraction experiments. A schematic view of the scattering experiment is shown in Fig. 3(a). In order to be consistent with the laboratory setup, the $x$-axis is taken along the [110] direction and defines the scattering plane together with the 
$z$-axis pointing along the [001] surface normal. The question now arises as to how an individual Ge-crystal among the thousands present in the array can be traced by the nano-focused X-ray beam. Fig. 3 indicates that this becomes increasingly difficult the taller the Ge-crystals become. Top view SEM images of 1 and $3 \mu \mathrm{m}$ high crystals grown on identical substrate patterns are shown in Fig. 3(b) and (c), respectively. They reflect the increasing width of the crystals due to the lateral expansion in the early stage of the growth $^{28}$. The total scattered intensity collected while scanning an X-ray beam across the sample under the azimuth angle appropriate for the $\mathrm{Ge}(115)$ reflection can be seen in the insets of Fig. 3(b) and (c). Evidently, individual crystals of this small height can be localized by the X-ray beam, making it possible to analyze their properties without any interference from nearest neighbors. We have shown in a previous publication that the elastic relaxation of the thermal strain in such short crystals happens by concave bending of the lattice planes ${ }^{322}$. This elastic lattice distortion rapidly dies out in the first few microns above the Ge/Si interface. For the taller crystals of interest here the elastically distorted region no longer contributes significantly to the scattering intensity. We are, however, confronted with the problem that such crystals no longer give rise to individual intensity maxima, as is evident from Fig. 3(d) for a height of $8 \mu \mathrm{m}$.

Fig. 4 demonstrates how single free-standing Ge-crystals were isolated by a combination of chemical etching and micromanipulation in order to permit measurements on individuals without any interference from nearest neighbors.

Now that free-standing crystals are available, it becomes possible to measure their tilt away from the exact [001] orientation. The real space image of a tilted crystal is shown in Fig. 5 along with the reciprocal space plane $Q_{x}, Q_{z}$ in the vicinity of a (115) reflection. The tilt 
angles $\chi_{x}$ and $\chi_{y}$ can be derived from the deviations of the maxima in the 3D RSMs measured near the top of the crystals from their exact nominal positions as follows. The way in which the position of the X-ray beam on a crystal can be identified is shown in Fig. 6. Here, after having received a signal from a particular crystal at some chosen scattering vector, the total scattering intensity is measured along the height of the crystal by translating the sample in the [110] direction. The tilt is then calculated from the RSM measured at the position $\mathrm{C}$ close to the top of the crystal. The tilts of three representative crystals (1,2 and 3) are summarized in the table shown in Fig. 5. The positions of the diffracted signals from these crystals are schematically illustrated in the RSM. Taking into account that in a laboratory measurement around $10^{5}$ crystals contribute to the diffracted intensity, all of which exhibit a slightly different tilt, the total diffracted signal is a superposition of very many peaks. This results in a broad peak around the Ge reflection resembling a mosaicity effect. It must be emphasized that individual Ge crystals do not exhibit any mosaicity at all, as will be shown below.

Basically, the tilt of tall Ge-crystals can arise from two sources. The first contribution is present only in asymmetric crystals, which are formed when trenches of unequal width are present, such as those in Fig. 1(b). In this case the tilt is caused by an asymmetric relaxation of the thermal $\operatorname{strain}^{28}$. The second contribution, present also in symmetric crystals, can be attributed to the dislocation network causing local tilts which do not average out on a microscopic scale. Taken together, these tilts account for most of the elongation of the diffraction peaks in the direction perpendicular to the scattering vector in Fig. 1 and

Fig. 2. 
Now that we can attribute the main source of the broadening of RSMs measured by a laboratory diffractometer to crystal tilts, the question remains to what extent the diffraction peaks are broadened further by the dislocations we know to be present at and in the vicinity of the interface. This information is also contained in the 3D RSMs measured along the height of a Ge crystal in Fig. 6. The inset of this figure shows cross sections along the $Q_{x}$ direction of reciprocal space through the intensity maxima of the $\mathrm{Ge}(115)$ reflection recorded at point A very close to the interface, point $\mathrm{B}$ farther away, and at point $\mathrm{C}$ near the top of the crystal. Evidently, there is a dramatic reduction of the FWHM of these curves with increasing distance from the $\mathrm{Ge} / \mathrm{Si}(001)$ interface which must be attributed to a diminishing contribution of dislocations as a function of height. The crystals hence become more and more perfect the farther away one moves from the heavily dislocated interface.

In order to see how far this increase of Ge-crystal perfection can go, we now compare the cross-sections of 3D RSMs of the Ge(115) reflection with the corresponding Bragg peak of the $\mathrm{Si}$ substrate which is known to be a perfect, defect-free crystal. Fig. 7(a) shows a perspective view of the 3D RSM in the reciprocal space region of interest, along with its projections into the $Q_{x} Q_{y}, Q_{x} Q_{z}$ and $Q_{y} Q_{z}$ planes. Magnified portions of the $Q_{x} Q_{z}$ projections are shown in Fig. 7(b) for the Si(115) reflection and in Fig. 7(c) for the Ge(115) reflection, together with cross-sections along $Q_{x}$ for both cases. Both the intense $\operatorname{Si}(115)$ and $\mathrm{Ge}(115)$ peaks are elongated due to the divergence of the X-ray beam. The larger, approximately round spot of lower intensity around the Ge(115) reflection stems from scattering by strained material in the trenches which was not completely removed by the hydrogen peroxide etching. The most striking result is obtained by comparing the cross-sections through the sharp $\mathrm{Si}(115)$ and Ge(115) peaks shown in Fig. 7(b) and (c). The 
width of the peaks is close to the pixel resolution of the $2 \mathrm{D}$ detector, which is why only very few data points can be seen. Within the resolution of the diffractometer we can state, however, that the perfection of the Ge-crystal is indistinguishable from that of a defect-free Si wafer.

\section{Summary}

We have carried out synchrotron X-ray nano-diffraction on individual species isolated from a space-filling array of tall Ge-crystals epitaxially grown onto deeply patterned $\mathrm{Si}$ substrates. Our results prove that far away from the heavily dislocated $\mathrm{Ge} / \mathrm{Si}$ interface the Ge-crystals have evolved into perfect single crystals within the resolution offered by the measurement. The peak broadening measured with ordinary high-resolution laboratory diffractometers, indicating crystal imperfection in the form of substantial mosaic spread, is explained in terms of a statistical distribution of net crystal tilts. The system studied here may therefore be considered as a model for defect-free lattice mismatched heteroepitaxy on patterned substrates, offering a wide range of potential applications, such as radiation and particle detectors, high-efficiency solar cells and light emitters, as well as power semiconductor devices.

\section{Acknowledgment}

Financial support by the Swiss federal program Nano-Tera through project NEXRAY is gratefully acknowledged. M.M. wishes to acknowledge the projects INGO LA 10010, R4I (CZ.1.07/2.4.00/17.0006), CEITEC (CZ.1.05/1.1.00/02.0068) and NANOE (CZ.1.07/2.3.00/20.0027). We thank B. Batlogg for his encouraging support, and the crew of the ID01 beamline at the European Synchrotron Radiation Facility (ESRF) for providing 
excellent user support for the scanning X-ray nano-diffraction experiments. We are indebted to A. Neels, T. Bandi for discussions about the laboratory high-resolution XRD experiments, to O. Caha for helpful considerations about diffraction theory, and to the FIRST Center for Micro- and Nanoscience of ETH Zürich for making available its infrastructure, as well as to Pilegrowth Tech Srl for providing their technical expertise. 


\section{References}

1. S. Lourdudoss, Current Opinion in Solid State \& Materials Science 16, 91 (2012)

2. D. Liang and J.E. Bowers, Nature Photonics 4, 511 (2010)

3. J. Michel, J. Liu, and L.C. Kimerling, Nature Photonics 4, 527 (2010)

4. R. Soref, Nature Photonics 4, 495 (2010)

5. J.W. Matthews, S. Mader, T.B. Light, J. Appl. Phys. 41, 3800 (1970)

6. J.W. Matthews, A.E. Blakeslee, S. Mader, Thin Solid Films 33, 253 (1976)

7. F.K. LeGoues, B.S. Meyerson, J.F. Morar, Phys. Rev. Lett. 66, 2903 (1991)

8. E.A. Fitzgerald E. A. Fitzgerald, Y.-H. Xie, M. L. Green, D. Brasen, A. R. Kortan, J. Michel, Y.-J Mii, and B. E. Weir, Appl. Phys. Lett. 59, 811 (1991)

9. S. Mantl, B. Holländer, R. Liedtke, S. Mesters, H.J. Herzog, H. Kibbel, T. Hackbarth, Nucl. Instr. Methods Phys. Res. B 147, 29 (1999)

10. H. Chen, L. W. Guo, Q. Cui, Q. Hu, Q. Huang, and J. M. Zhou, J. Appl. Phys. 79, 1167 (1996)

11. E. Kasper, K. Lyutovich, M. Bauer, M. Oehme, Thin Solid Films 396, 319 (1998)

12. P. D. Hodson, P. Kightley, R. C. Goodfellow, T. B. Joyce, J. R. Riffat, R. R. Bradley and R. J. M. Griffiths Semicond. Sci. Technol. 3, 715 (1988)

13. E.A. Fitzgerald, N. Chand, J. Electron. Mat. 20, 839 (1991)

14. For a review of early work, see also A. Madhukar, Thin Solid Films 231, 8 (1993)

15. T. Soga, S. Sakai, M. Umeno, S. Hattori, Jpn. J. Appl. Phys. 26, 252 (1986)

16. M.R. Goulding, Mat. Sci. Eng. B17, 47 (1993)

17. H. -C. Luan, D. R. Lim, K. K. Lee, K. M. Chen, J. G. Sandland, K. Wada, and L. C. Kimerling , Appl. Phys. Lett. 75, 2909 (1999) 
18. T. A. Langdo, C. W. Leitz, M. T. Currie, E. A. Fitzgerald, A. Lochtefeld, and D. A. Antoniadis, Appl. Phys. Lett. 76, 3700 (2000)

19. J.-S. Park, J. Bai, M. Curtin, B. Adekore, M. Carroll, and A. Lochtefeld , Appl. Phys. Lett. 90, 052113 (2007)

20. G. Wang, E. Rosseel, R. Loo, P. Favia, H. Bender, M. Caymax, M. M. Heyns, and W. Vandervorst, Appl. Phys. Lett. 96, 111903 (2010)

21. E.P. Kvam, D. M. Maher and C. J. Humphreys, J. Mater. Res. 5, 1900 (1990)

22. G.R. Booker, J.M. Titchmarsh, J. Fletcher, D.B. Darby, M. Hockly, M. Al-Jassim, J. Cryst. Growth 45, 407 (1978)

23. H. Klapper, Mat. Chem. Phys. 66, 101 (2000)

24. J. Bai, J.-S. Park, Z. Cheng, M. Curtin, B. Adekore, M. Carroll, A. Lochtefeld, and M. Dudley, Appl Phys. Lett. 90, 101902 (2007)

25. A. Marzegalli, F. Isa, H. Groiss, E. Müller, C. V. Falub, A. G. Taboada, P. Niedermann, G. Isella, F. Schäffler, F. Montalenti, H. von Känel, L. Miglio, Adv. Mat. 25(32):4408-12 (2013)

26. V. K. Yang, M. Groenert, C. W. Leitz, A. J. Pitera, M. T. Currie, and E. A. Fitzgerald J. Appl. Phys. 93, 3859 (2003)

27. M. Sakai, T. Egawa, M. Hao, H. Ishikawa, Jpn. J. Appl. Phys. 43, 8019 (2004)

28. C.V. Falub, H. von Känel, F. Isa, R. Bergamaschini, A. Marzegalli, D. Chrastina, E. Müller, P. Niedermann, L. Miglio, Science 335, 1330 (2012)

29. C. Rosenblad, H.R. Deller, A. Dommann, T. Meyer, P. Schroeter, and H. von Känel, J. Vac. Sci. Technol. A 16, 2785 (1998) 
30. D. Chrastina, G. M. Vanacore, M. Bollani, P. Boye, S. Schöder, M. Burghammer, R. Sordan, G. Isella, M. Zani, and A. Tagliaferri, Nanotechnology 23, 155702 (2012)

31. D.C. Houghton, D.D. Perovic, J.M. Baribeau, and G.C. Weatherly, J. Appl. Phys. 67, $1850(\mathbf{1 9 9 0})$

32. C.V. Falub, M. Meduňa, D. Chrastina, F. Isa, A. Marzegalli, T. Kreiliger, A. G. Taboada, G. Isella, L. Miglio, A. Dommann, and Hans von Känel, Sci. Rpts. 3, 2276 (2013) 
a
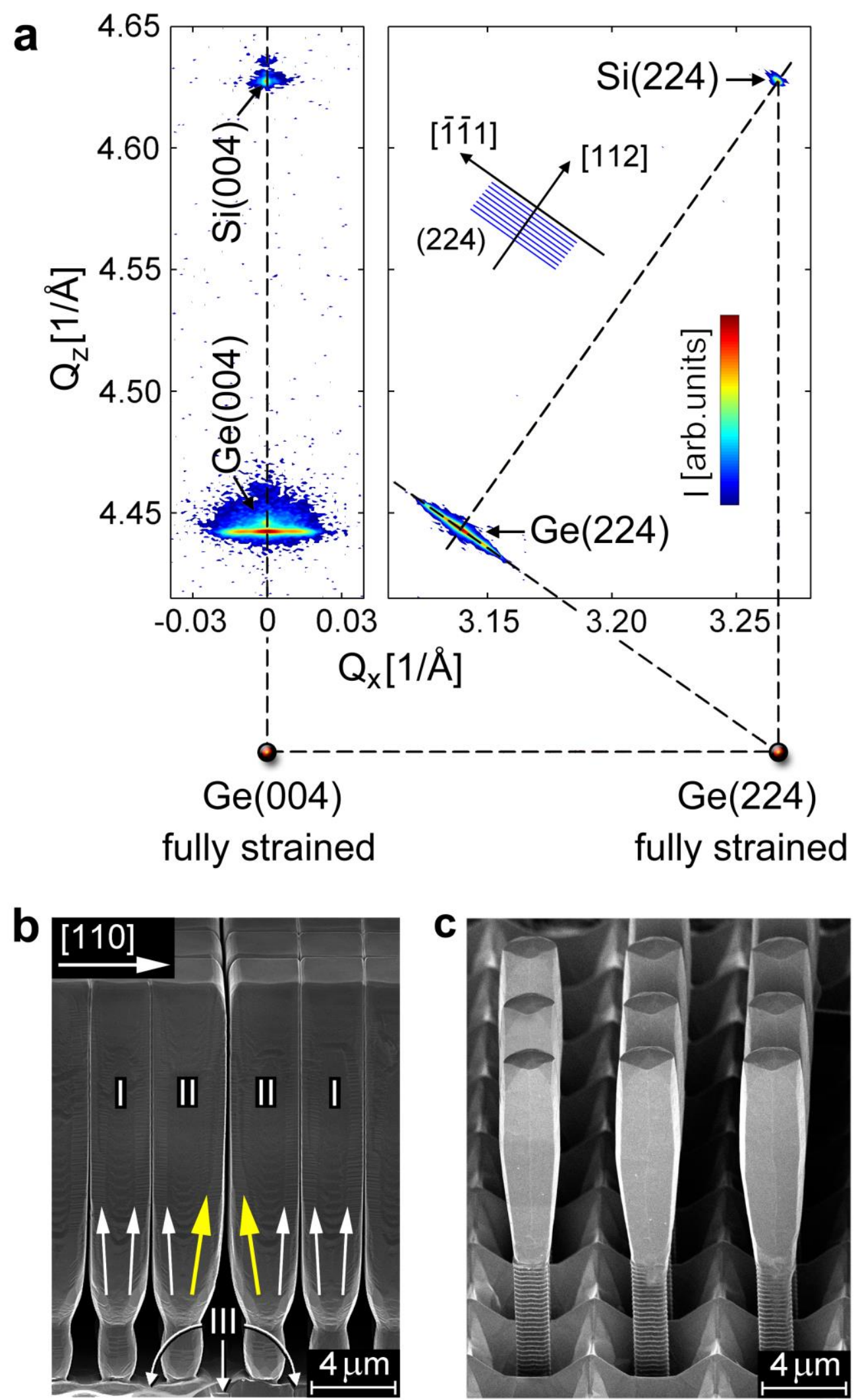
Fig. 1: (a) RSMs recorded with the laboratory diffractometer in the vicinity of the $\mathrm{Si}(004)$ and $\mathrm{Si}(224)$ reflections on a space filling array of $16 \mu \mathrm{m}$ tall Ge crystals on top of $2 \mu \mathrm{m}$ wide Si pillars. The contour plots are represented on a logarithmic scale. SEM images of typical arrays of faceted Ge crystals are shown as grown and after etching in a $\mathrm{H}_{2} \mathrm{O}_{2}$ solution in (b) and (c), respectively.

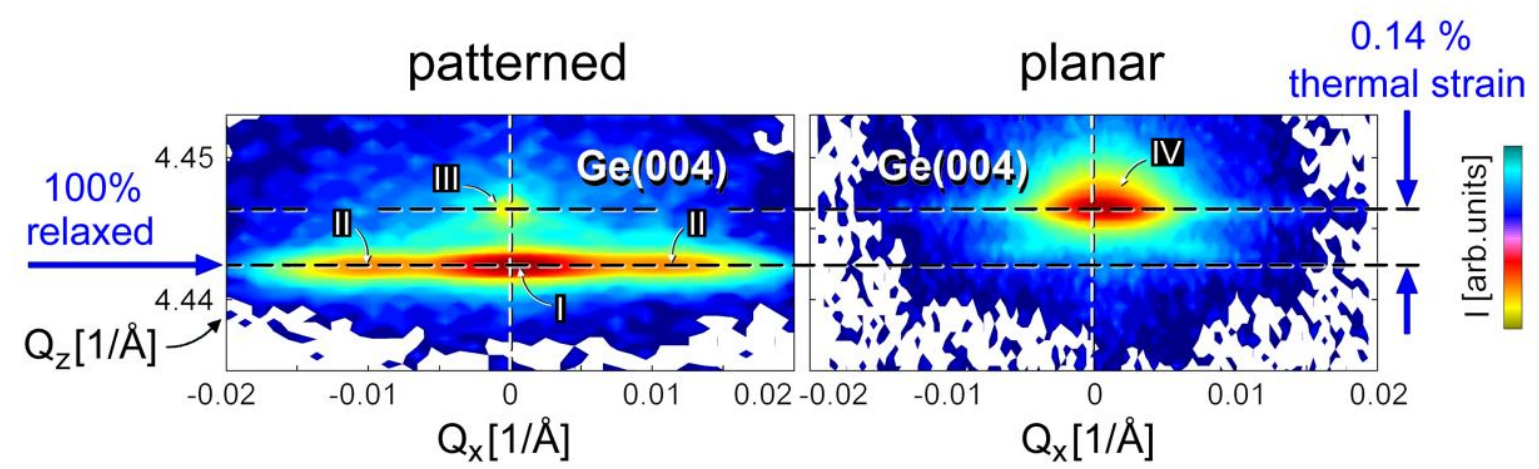

Fig. 2: Magnified region of the RSM of Fig. 1 around the Ge(004) reflection (left) and comparison with the corresponding RSM of a planar Ge layer of the same thickness (right). The Roman numerals describe different regions in real space indicated in Fig. 1. 

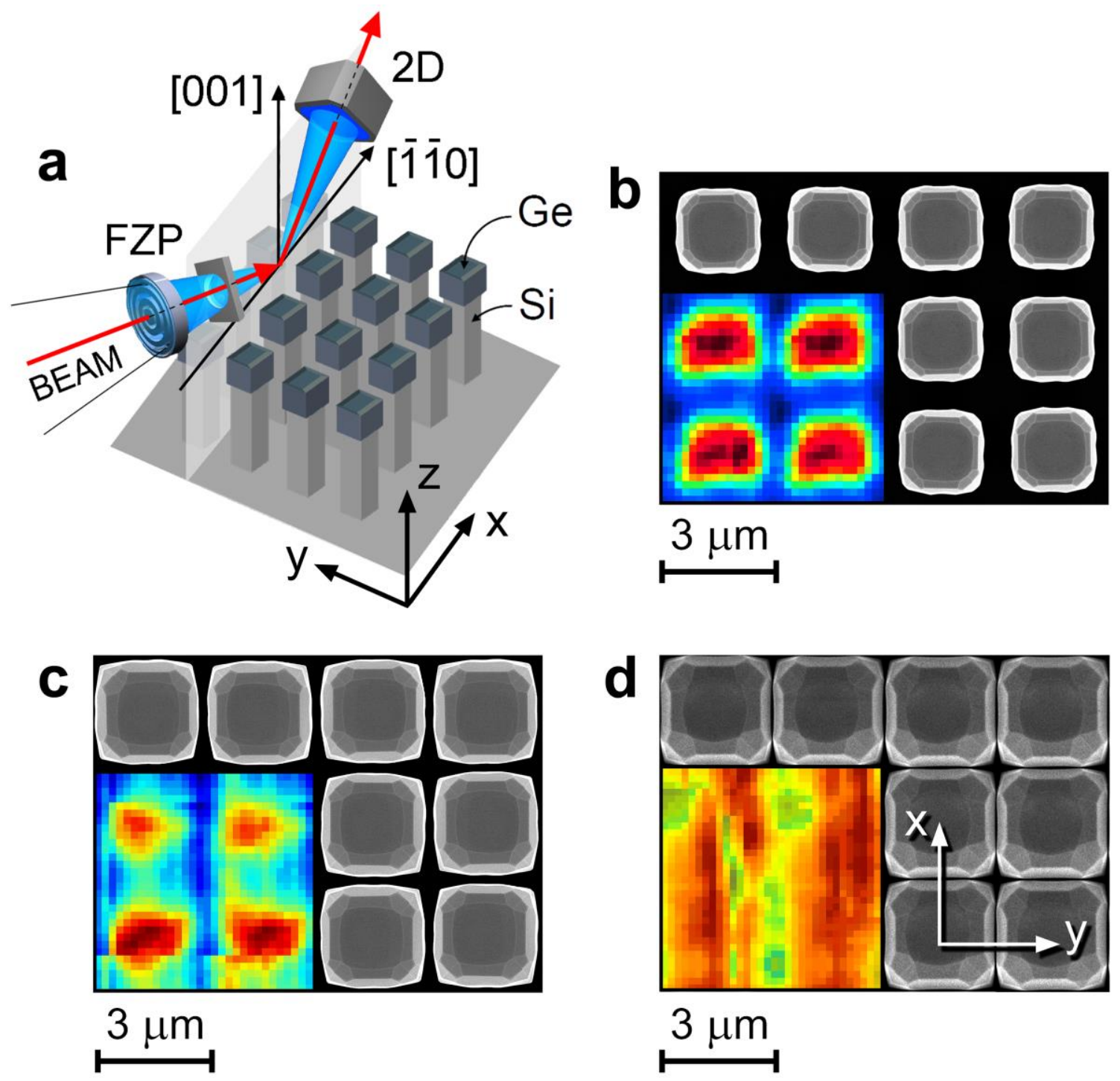

Fig. 3: (a) Scattering geometry used for the nano-diffraction experiments. The $x$-axis is taken along the [110] direction forming the intersection between the substrate and the scattering plane, the $y$-axis is perpendicular to the scattering plane, and the $z$-axis points along the [001] surface normal. (b)-(d) show top SEM views of 1, 3 and $8 \mu \mathrm{m}$ tall Ge-crystals, respectively, along with the total scattering intensity collected around the Ge(115) reflection as the X-ray beam is scanned across the crystal arrays. 


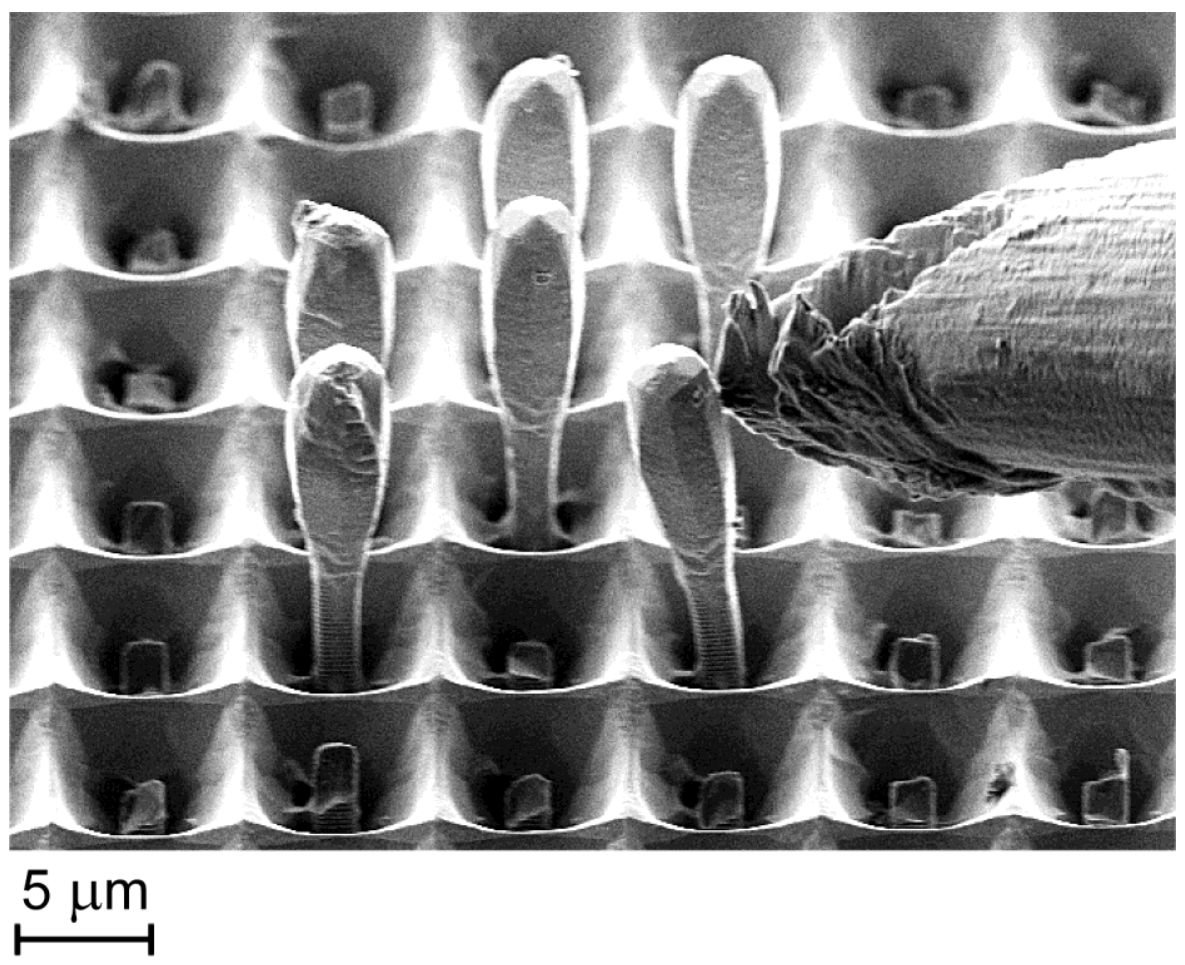

Fig. 4: Example of sample preparation for X-ray nano-diffraction experiments on isolated Ge-crystals. The sample is first etched in $\mathrm{H}_{2} \mathrm{O}_{2}$ (see also Fig. 1(c)), to increase the gaps between nearest neighbours. It is then transferred into an SEM equipped with micromanipulators, by means of which material can be removed, resulting in free standing crystals suitable for measurements without any interference form nearest neighbours. 


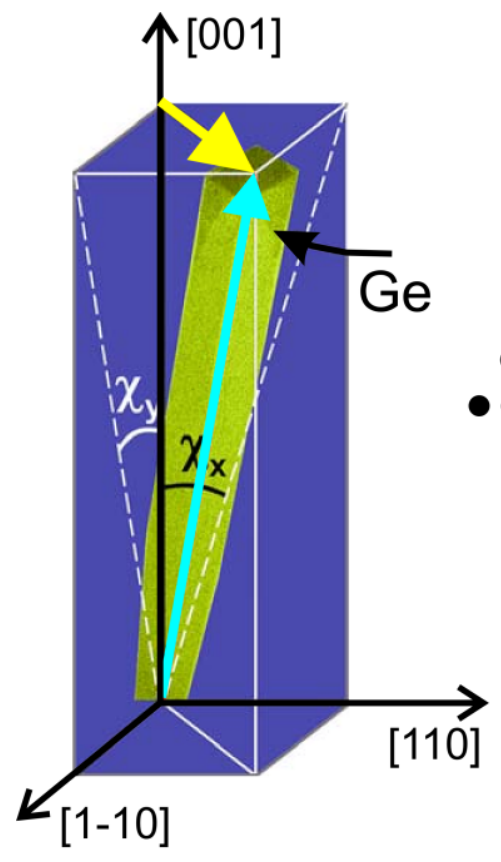

Real space

\begin{tabular}{l|cc}
\hline Crystal & $\chi_{x}\left(^{\circ}\right)$ & $\chi_{y}\left(^{\circ}\right)$ \\
\hline$\bullet 1$ & $-0.023 \pm 0.006$ & $0.033 \pm 0.005$ \\
$\circ 2$ & $-0.006 \pm 0.001$ & $0.068 \pm 0.001$ \\
$\circ 3$ & $0.012 \pm 0.001$ & $0.023 \pm 0.001$ \\
\hline
\end{tabular}

- nominal peak, $\chi_{x, y}=0$, relaxed $\mathrm{Ge} \quad$ signal superimposed - $\bigcirc$ peaks from individual tilted crystals from all Ge crystals
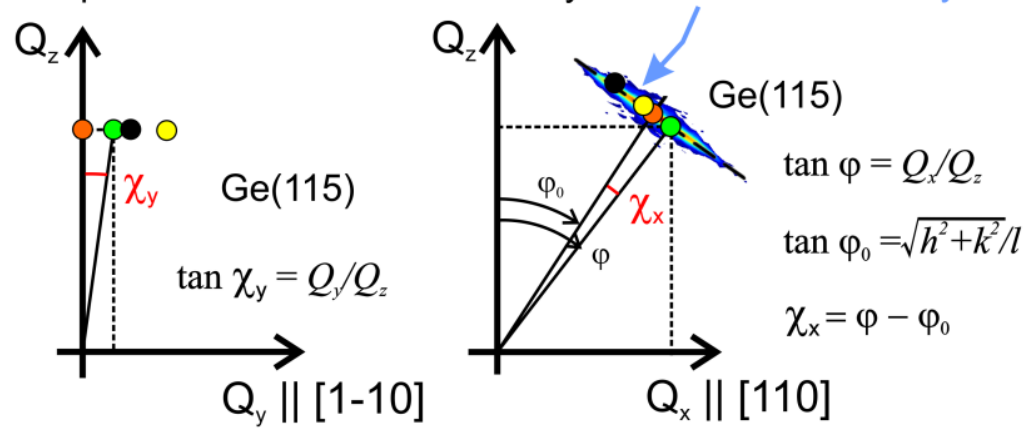

Reciprocal space

Fig. 5: Measurement of the tilt of tall Ge-crystals. The maxima of the RSMs of asymmetric reflections measured on top of the crystals are rotated by the tilt angles $\chi_{x}$ and $\chi_{y}$ relative to their nominal positions corresponding to perfect alignment along [001]. The tilt angles of three representative crystals are given in the table and indicated by spots in the RSM. Averaging over thousands of crystals with individual tilts results in the broadened peaks visible also in Fig. 1 and Fig. 2. 


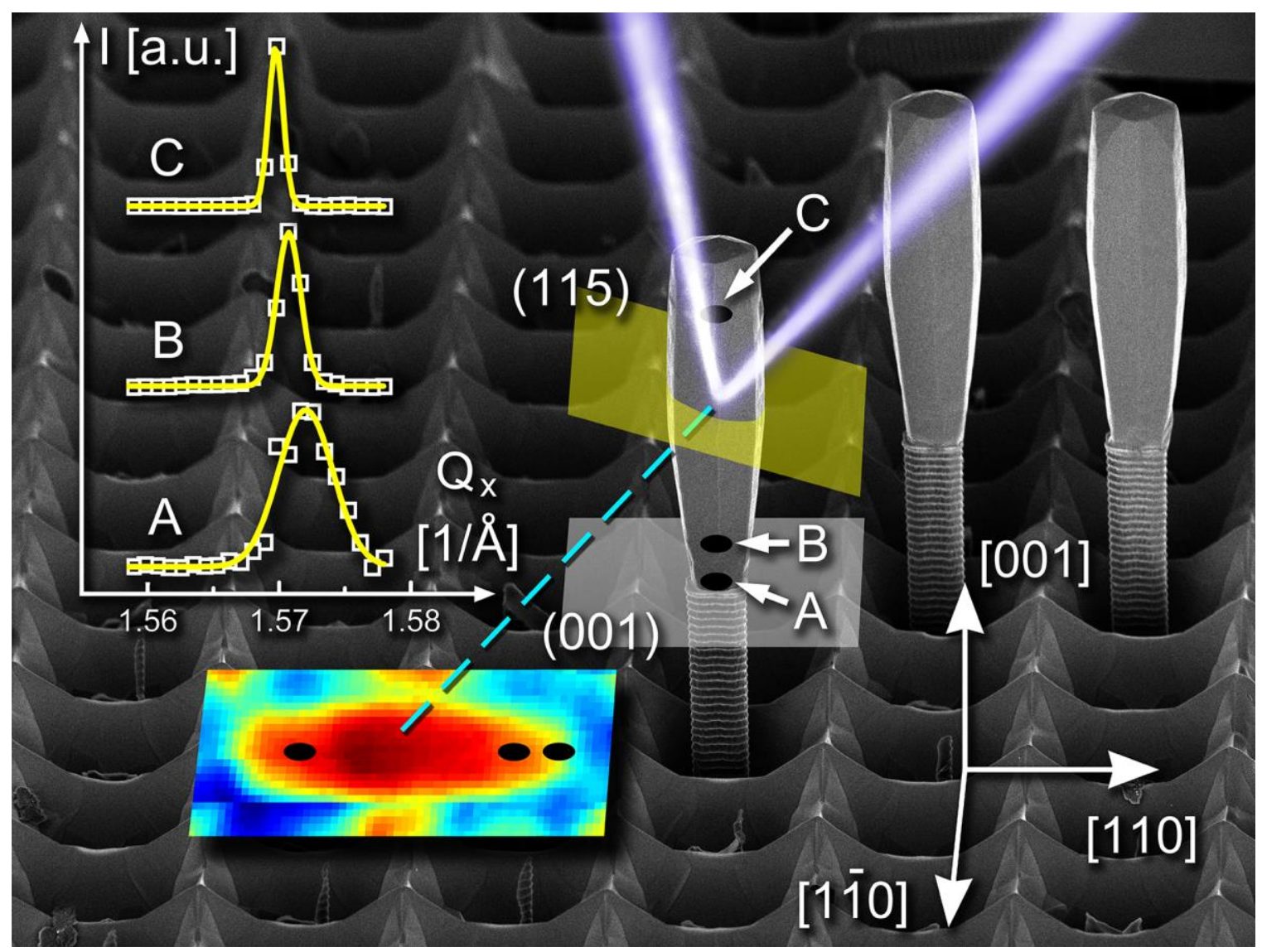

Fig. 6: X-ray nano-diffraction from $16 \mu \mathrm{m}$ tall, free-standing Ge-crystals. The total scattering intensity measured as the sample is translated along [110], allows the identification of vertical position from which the scattering originates. This information is then used to measure RSMs as a function of the distance from the $\mathrm{Ge} / \mathrm{Si}[001]$ interface. Examples of $Q_{x}$ cross-sections through such RSMs, taken at positions A, B and C, are shown in the inset. 

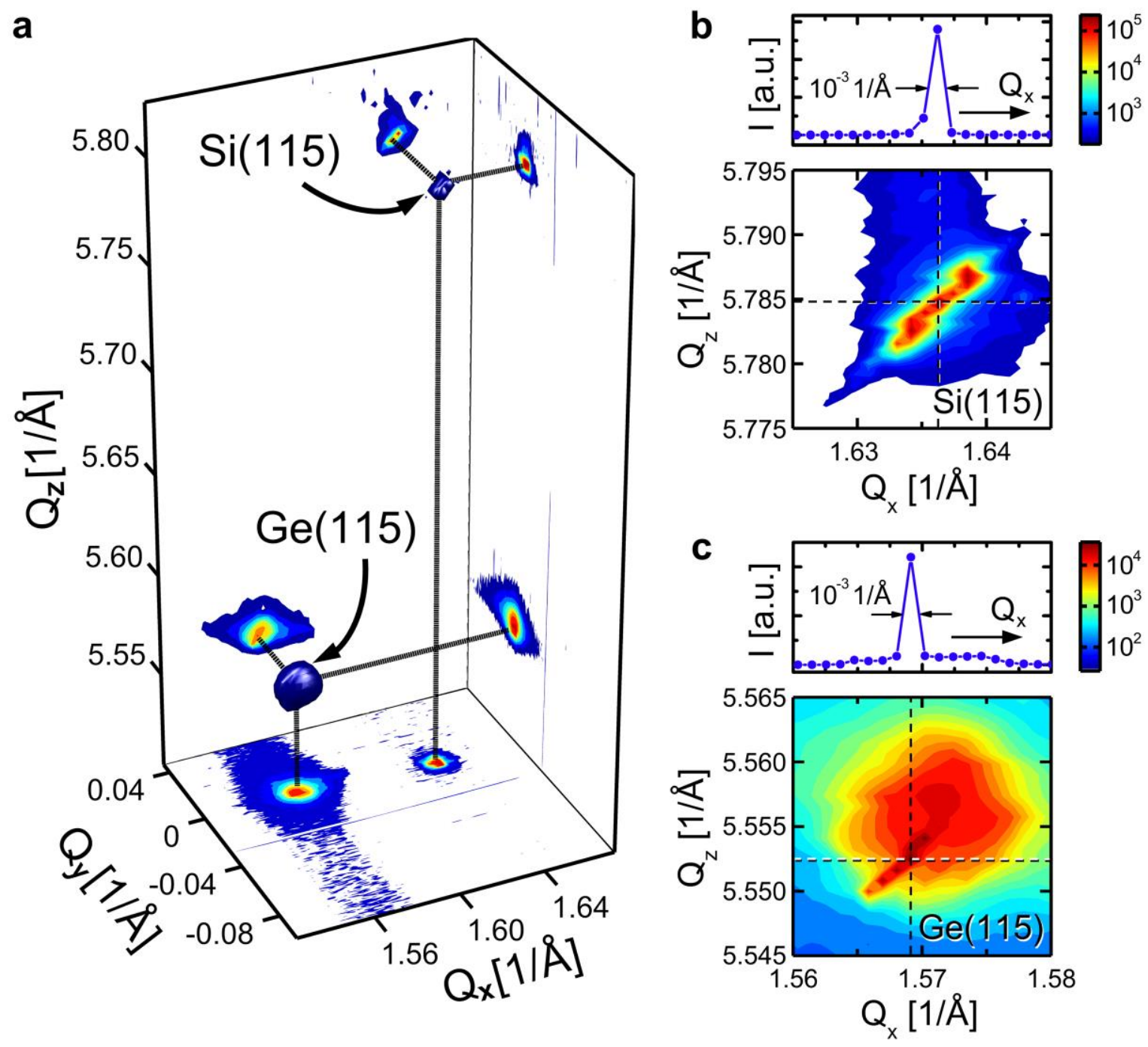

Fig. 7: (a) 3D RSMs in the vicinity of the $\mathrm{Ge}(115)$ and $\mathrm{Si}(115)$ reflections, together with their projections into the $Q_{x} Q_{y}, Q_{x} Q_{z}$ and $Q_{y} Q_{z}$ planes. (b) Magnified region of the $Q_{x} Q_{z}$ projection around the $\operatorname{Si}(115)$ reflection along with its cross-section along $Q_{x}$. (c) Magnified region of the $Q_{x} Q_{z}$ projection around the $\operatorname{Ge}(115)$ reflection along with its cross-section along $Q_{x}$. The elongation of the spots in (b) and (c) is due to the divergence of the focused X-ray beam. 